\title{
Topologically distinct critical theories emerging from the bulk entanglement spectrum of integer quantum Hall states on a lattice
}

\author{
Qiong Zhu ${ }^{1}$, Xin $\mathrm{Wan}^{1}$, and Guang-Ming Zhang ${ }^{2,3}$ \\ ${ }^{1}$ Zhejiang Institute of Modern Physics, Zhejiang University, Hangzhou 310027, China \\ ${ }^{2}$ State Key Laboratory of Low-Dimensional Quantum Physics and \\ Department of Physics, Tsinghua University, Beijing 100084, China \\ ${ }^{3}$ Collaborative Innovation Center of Quantum Matter, Beijing, China
}

(Dated: September 27, 2021)

\begin{abstract}
The critical theories for the topological phase transitions of integer quantum Hall states to a trivial insulating state with the same symmetry can be obtained by calculating the ground state entanglement spectrum under a symmetric checkerboard bipartition. In contrast to the gapless edge excitations under the left-right bipartition, a quantum network with bulk gapless excitations naturally emerges without fine tuning. On a large finite lattice, the resulting critical theory for the $\nu=1$ state is the $(2+1)$ dimensional relativistic quantum field theory characterized by a single Dirac cone spectrum and a pair of fractionalized zero-energy states, while for the $\nu=2$ state the critical theory exhibits a parabolic spectrum and no sign of fractionalization in the zero-energy states. A triangular correspondence is established among the bulk topological theory, gapless edge theory, and the critical theory via the ground state entanglement spectrum.

PACS numbers: 73.43.Cd, 73.43.Nq, 03.67.Mn
\end{abstract}

Introduction.- The integer quantum Hall (IQH) effect has been well studied in various two-dimensional (2D) electron systems, including the experimental observation in graphene 1, 2]. When the time reversal symmetry is broken, the 2D electron systems are characterized by a topological quantum number known as the Chern number 3], which can be measured experimentally as the quantized Hall conductance with high precision [4]. In 1988, Haldane [5] proposed a famous honeycomb lattice model for the IQH effect, which allows the access to the phase transition between the pure Hall state $(\nu=1)$ and its trivial insulating state $(\nu=0)$ through tuning the time-reversal symmetry or inversion symmetry breaking parameters. Such a transition is a prototype of topological quantum phase transition. Unlike the LandauGinzberg-Wilson paradigm for the symmetry breaking phase transitions, the quantum criticality is characterized by the Dirac spectrum with the parity anomaly and undoubled chiral fermions in the lattice realization of the $(2+1)$ D relativistic quantum field theory [5, 6]. Moreover, interesting features can arise in the phase transitions for the $\nu>1$ IQH states, e.g., charge carriers with the parabolic energy spectrum in pristine bilayer graphene exhibit distinct features with a double-step transition [7]. Therefore, a systematic method is called for to study the critical properties of the $2 \mathrm{D}$ topological phases.

It is generally believed that the essential information of a topological phase is encoded in its ground state wave function. $\mathrm{Li}$ and Haldane [8] introduced entanglement spectrum as the collection of eigenvalues of the reduced density matrix under a left-right bipartition, and found that the low-lying spectrum bears a remarkable similarity to the physical edge spectrum of the topological state 9 12]. A further step is to ask how one can extract the bulk critical properties for the transition from the topo- logical phase to its trivial insulating phase with the same symmetry, and what the relationship is between the corresponding gapless edge theory and the bulk critical theory.

In fact, the critical theory can be regarded as the "domain wall" between the topological phase and its trivial insulating phase in the parameter space, hence realizing delocalized boundary excitations of the non-trivial topological phase 13. To reveal such a non-trivial quantum criticality, appropriated boundary conditions are needed. For the IQH transitions, the domains of trivial and nontrivial phases described by a Dirac Hamiltonian with respective positive and negative masses [5, 6] must be adjacent to each other in the model for the critical theory. Accordingly, the edge modes along the boundaries of the domains form a percolating network. As Haldane pointed out [5], along the critical line between the trivial and topological phases, one expects a low-lying massless Dirac spectrum. Hsieh and Fu [14] argued that a symmetric extended bipartition of the system into two subsystems as long as the size of the sublattice unit cell is larger than the correlation length of the topological phase can realize such boundary conditions.

According to Ref. [14], the bulk entanglement spectrum of the IQH state with $\nu=1$ (or odd integers) is gapless, while that of a state characterized by an even Chern number may be gapped. This is counterintuitive because the conclusion suggests that the bulk entanglement spectrum of a $\mathbb{Z}$ topological insulator is characterized by a $\mathbb{Z}_{2}$ index; in other words, the bulk entanglement spectrum and the edge theory do not match. Should there, then, be a one-to-one correspondence at all between the edge structure of a topological phase and its critical bulk entanglement spectrum? Does the emergent symmetries at the critical point play a role for the mismatch? In 
this Letter, we answer these questions by analyzing the bulk entanglement spectrum in a lattice model of IQH states with $\nu=1$ and, more importantly, $\nu>1$. We show that the critical theory for the $\nu=1$ state is the $(2+1)$-dimensional relativistic quantum field theory characterized by a single Dirac cone spectrum and a pair of fractionalized zero-energy states. For the $\nu=2$ state, the bulk entanglement spectrum exhibits a quadratic band crossing. The spectral difference resembles vividly that between monolayer graphene [1, 2, 15] and pristine bilayer graphene [7, 16]. In general, we find that the Chern index of the topological phase is in one-to-one correspondence with the Berry flux for the ground state wave function of the entanglement Hamiltonian. Extending the bulkedge correspondence of a topological phase, we propose a generic triangular correspondence among the bulk topological theory, gapless edge excitations, and the critical theory as revealed by the bulk entanglement spectrum.

Model and method.- We consider a tight-binding Hamiltonian on a 2D square lattice in the presence of a perpendicular magnetic field,

$$
H=-\sum_{\langle i j\rangle}\left(e^{i \theta_{i j}} c_{i}^{\dagger} c_{j}+\text { h.c. }\right)
$$

where the total phase that an electron picks up when moving around a plaquette

$$
\sum_{\square} \theta_{i j}=2 \pi \frac{\phi}{\phi_{0}}
$$

is given by the magnetic flux $\phi$ per unit cell, in units of magnetic flux quantum $\phi_{0}=h c / e$. The energy spectrum of the system exhibits a self-similar pattern, known as the Hofstadter butterfly [17]. We first focus on the case of $\phi / \phi_{0}=1 / 3$, at which the tight-binding band is split into three magnetic subbands, which carry Chern numbers $\nu=1,-2$, and 1, respectively. Figure 1(a) shows the density of states from a $300 \times 300$ square lattice. With the open boundary condition in the $y$ direction, the corresponding spectrum includes chiral edge states in the two band gaps, as illustrated in Fig. 1(b). Actually the tight-binding Hamiltonian can describe a lattice realization of the Landau levels and the subsequent IQH transitions. The topological phases belong to the class A in the classification of noninteracting topological insulators/superconductors [18]. The time-reversal symmetry is explicitly broken by the external magnetic flux, which determines the propagation direction of the chiral edge modes.

The presence of the chiral edge modes in the topological system is an example of the so-called bulk-edge correspondence. It also manifests at the artificial boundary in the study of quantum entanglement [8]. After we fill up the lowest magnetic subband in the case of $\phi=\phi_{0} / 3$, the Fermi energy lies in the spectral gap and the system is in the $\nu=1 \mathrm{IQH}$ phase. If we cut a square $\mathrm{A}$ in the
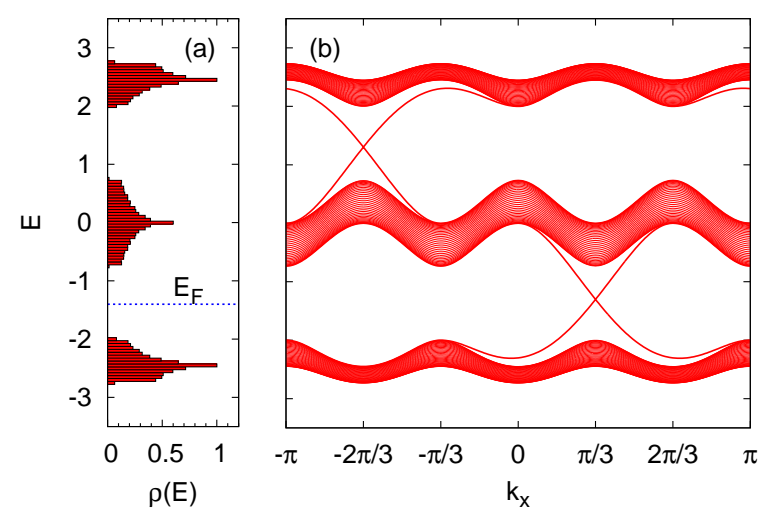

FIG. 1: (Color online) (a) Density of states of the tightbinding $300 \times 300$ lattice model with periodic boundary conditions. (b) Corresponding dispersion curves with open boundary condition in $y$-direction only.

bulk of the system and trace out all the degrees of freedom in area B outside the square in the density matrix of the ground state $\Psi_{0}$, the spectrum of the resulting reduced density matrix $\rho_{A}=\operatorname{Tr}_{B}\left(\left|\Psi_{0}\right\rangle\left\langle\Psi_{0}\right|\right)$ resembles the energy spectrum of a chiral Fermi liquid, which is described by a $(1+1) \mathrm{D}$ chiral massless Dirac fermion field theory. Heuristically, we can represent such a bipartition by a directed loop surrounding $\mathrm{A}$.

The technique on computing the spectrum of the reduced density matrix for non-interacting systems has been well documented in the literature [19]. The key lies in the computation of the correlation matrix $g_{i j}=\left\langle c_{i}^{+} c_{j}\right\rangle$ in the ground state, where $i, j$ denote the lattice sites in the subsystem A. The correlation matrix is isospectral to the reduced density matrix $\rho_{A} \equiv e^{-H_{E}}$ or the entanglement Hamiltonian $H_{E}$. For a non-interacting electron system, the entanglement Hamiltonian takes a quadratic form

$$
H_{E}=\sum_{n, m} h_{n m} c_{n}^{+} c_{m} .
$$

More precisely, $g_{i j}$ encodes the Fermi-Dirac distribution function for the eigenstates of $H_{E}$ such that the eigenvalues of $g_{i j}$ are expressed as $\eta_{l}=1 /\left(e^{\varepsilon_{l}}+1\right)$, in which $\varepsilon_{l}$ s are the eigenvalues of $H_{E}$, or the energy levels of the ground state entanglement spectrum. As usual, the states with negative $\varepsilon_{l}$ are occupied.

Emergence of a single Dirac cone.- To reveal the information beyond the edge properties of the IQH $\nu=1$ state, we implement the symmetric checkerboard bipartition of a finite lattice 14], as illustrated in Fig. 2(a). Heuristically, the remaining half of the square blocks supports chiral edge modes (one per block) represented by directed loops, which form a regular lattice with the unit cell twice the size of the block and rotated by $\pi / 4$. With quantum tunneling at the block corners, the loops coa- 


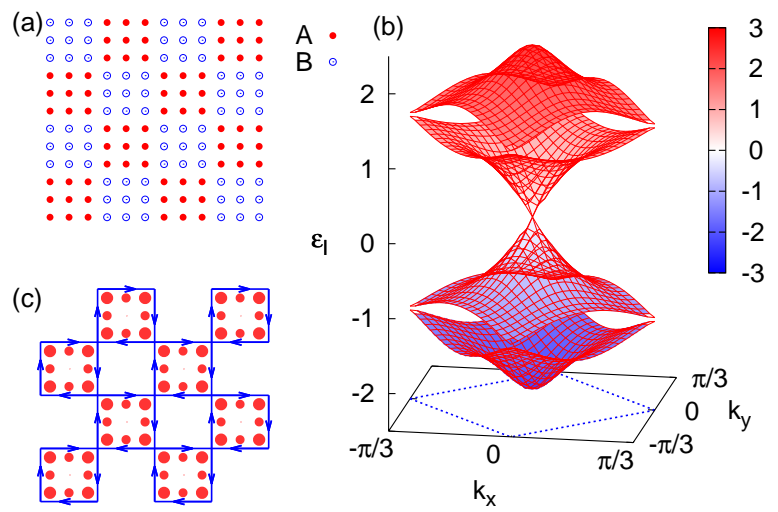

FIG. 2: (Color online) (a) Symmetric checkerboard bipartition illustrated in a $12 \times 12$ lattice. (b) Spectrum of the entanglement Hamiltonian $H_{E}$. For the clarity of the Dirac spectrum centered at $k=(0,0)$ and $\varepsilon_{l}=0$, we only plot the four bands with energies closest to zero. The Brillouin zone corresponding to the checkerboard lattice is illustrated by the blue square. (c) The total probability density of the two zeroenergy states in the finite $12 \times 12$ lattice, whose chiral edgestate nature is represented by the directed loops. The area of each dot is proportional to the local probability density.

lesce into a regular network. If we further assign random tunneling amplitudes at the corners, we will obtain the Chalker-Cottington network model [20], which can describe the generic IQH plateau transition.

Figure 2(b) plots the spectrum of the entanglement Hamiltonian $H_{E}$ for a large square lattice with a linear size $L=300$. Here, we choose each block in part A or $\mathrm{B}$ to include $3 \times 3$ sites, or a total magnetic flux $3 \phi_{0}$. For clarity, we only plot the four bands (out of nine) closest to zero in the entanglement spectrum. The most remarkable feature of the spectrum is the single Dirac cone appearing at the Brillouin zone center. The emergent entanglement spectrum hence differs qualitatively from the energy spectrum of the original lattice model. For comparison, we note that the Hamiltonian (11) with $\phi=\phi_{0} / 2$ has two Dirac cones in its energy spectrum that usually appears in lattice realizations of the $(2+1) \mathrm{D}$ relativistic quantum field theories. The defeat of the fermion doubling here is rooted both in the broken time-reversal symmetry of the original lattice model and in the broken 2D parity symmetry due to the checkerboard bipartition. Accordingly, the bipartition generates a non-interacting Hamiltonian $H_{E}$ on the non-simply connected 2D sublattice A with long-range $(\sim 1 / r)$ complex hopping amplitudes.

At the Dirac cone vertex, there always exist two degenerate zero-energy states, regardless of the size of the lattice system and the block size for the checkerboard bipartition; the Kramers degeneracy is the consequence of the emergent time-reversal symmetry of the low-energy effective Dirac Hamiltonian $H_{1}=\alpha \sigma \cdot \mathbf{p}[\underline{6}]$. We plot
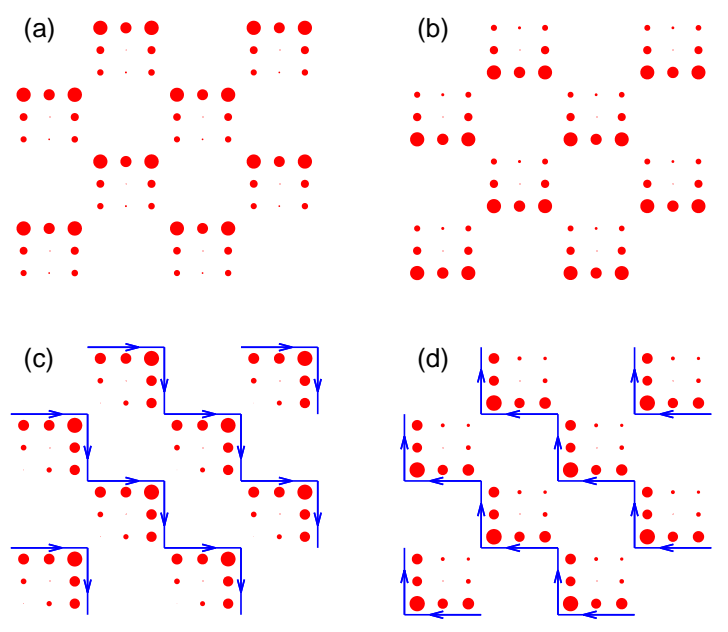

FIG. 3: (Color online) (a) and (b) The probability densities (proportional to the area of the dots) of the two zero-energy states (we only show a $12 \times 12$ sector for clarity). The probability is significant on half of the edge sites from each block only. (c) and (d) The probability densities of the two zeroenergy states in (a) and (b) after a rotation of $\pi / 8$ in their spinor representation. The sites with significant probability density follow chiral stairs either up or down.

the total probability density of the zero-energy states in Fig. 2(c) by filled circles. In each $3 \times 3$ block the density retains the point-group symmetry of the lattice and the center site in each block has zero density. This is a striking manifestation of the constituting edge mode within each block. With the directed loops attached around the blocks, we emphasize the emergence of the disorderfree Chalker-Cottington network picture[20], which can be mapped to the Dirac Hamiltonian in 2D system [21]. In fact, the eigenstates of the whole Dirac cone are linear combinations of the edge modes of individual blocks.

Fractionalization of zero-energy states.- The single Dirac cone structure in the entanglement spectrum also demonstrates that the low-energy effective Hamiltonian $H_{1}$ has a particle-hole symmetry, which is not present in the original lattice model [5, 6]. A further consequence is the charge fractionalization due to the parity anomaly of the $(2+1)$ D effective field theory [22]. We have carefully studied this two-dimensional space spanned by the zeroenergy states at the Dirac cone vertex by plotting their separate probability densities. As illustrated in Fig. 3(a) and (b), the fractionalization manifests in a way that either state is formed by the significant occupations on only half of the edge sites in each block. The fractionalization cannot be removed by any linear combinations of the two states, which only rotate the probability density around the edge in each block simultaneously.

The fractionalization of the edge degrees of freedom of each block is consistent with the fact that the Dirac cone emerges at the center of the Brillouin zone, hence 
representing the long-wavelength collective behavior of the system. By a $\pi / 8$-rotation of the zero-energy states in their spinor representation, the probability densities rotate $\pi / 4$ in the real space along the edge of each block, and we effectively fractionalize the network model as in Fig. 3(c) and (d). One state has the probability density essentially on the edges marked by right and down arrows - the right mover, while the other by left and up ones - the left mover. They form chiral, coherent, and penetrating paths across the system along the diagonal direction. The features of the fractionalization become more prominent as we increase the block size in each sublattice, which allows a cleaner separation of the bulk and the edge of each block. According to the Jackiw argument 22], the charge conjugation symmetry leads to a quantum Hall effect at $\nu=1 / 2$ with the zero-energy states filled and at $\nu=-1 / 2$ with them empty.

Topologically distinct transitions.- The low-lying massless spectrum of the undoubled chiral fermions and the parity anomaly confirms that the phase transition from the $\sigma_{x y}=e^{2} / h \mathrm{IQH}$ state to an insulator can emerge from the entanglement spectrum of the ground state wave function in the non-trivial phase. In unspecific words, we can unveil the full information of the gapless critical point from the wave function on the topological side. To support the general statement, we continue to explore the emergent physics under the checkerboard bipartition for the IQH state with $\sigma_{x y}=2 e^{2} / h$.

In Figure 4(a), we plot the entanglement spectrum in a $\phi=\phi_{0} / 9$ system with the lowest two magnetic subbands filled, which is a topological state with $\sigma_{x y}=2 e^{2} / h$. In contrast to the Dirac cone spectrum in the $\sigma_{x y}=e^{2} / h$ case, we find undoubled chiral fermions with a parabolic dispersion associated with a Berry flux of $2 \pi$. In addition, a pair of adjacent bands [red lines in Fig. 4(a)] is also visible with a gap opened at the Brillouin center. The band structure, together with the earlier $\sigma_{x y}=e^{2} / h$ case, reminds us of the spectral difference between monolayer and pristine bilayer graphene [15], except that the graphene systems have two valleys at the two inequivalent corners of their hexagonal Brillouin zone. In our case, the parabolic low-energy spectrum emerges at the zone center without fine tuning. The four bands can be regarded as resulting from the reconstruction of the two Dirac cones which one may naively expect to appear at the zone center, if one asserts that no quantum entanglement exists between states in different subbands separated by a spectral gap. The existence of the quadratic band-crossing point is allowed due to the emergent timereversal symmetry and the $C_{4}$ lattice symmetry in the original model [23]. We further find that, like graphene, the distinction of the $\sigma_{x y}=e^{2} / h$ and $2 e^{2} / h$ cases lies in the Berry flux ( $\pi$ and $2 \pi$, respectively) of the chiral fermions 16. Therefore, the emergent spectrum describes a double-step transition from the $\sigma_{x y}=2 e^{2} / h$ $\mathrm{IQH}$ state to the corresponding $\sigma_{x y}=0$ state, much like
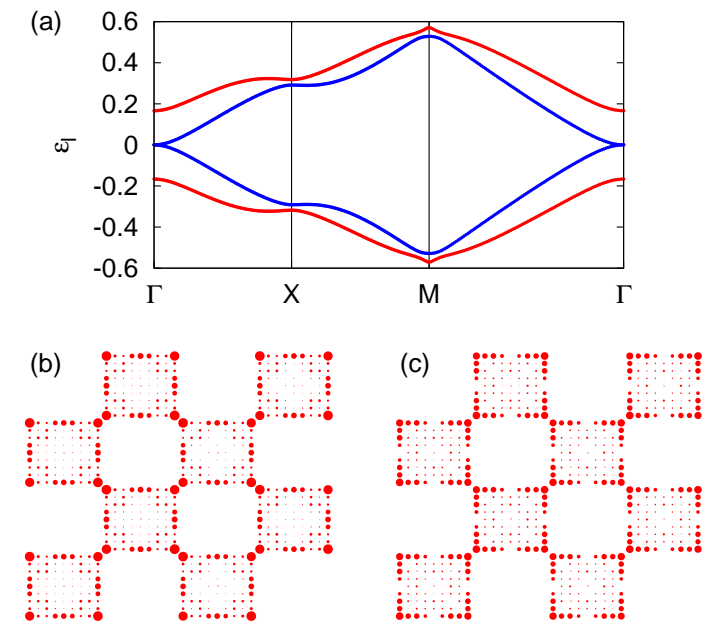

FIG. 4: (Color online) (a) Dispersion curves of the entanglement spectrum from the checkerboard bipartition (in $9 \times 9$ blocks) with $\phi=\phi_{0} / 9$ and two magnetic subbands filled. (b) and (c) The probability densities (proportional to the area of the dots) of the two zero-energy states.

the double-step IQH transition in bilayer graphene 7 ].

The fractionalization of the zero-energy states is the hallmark of the $(2+1) \mathrm{D}$ relativistic quantum field theory, hence ought to be absent in the $\sigma_{x y}=2 e^{2} / h$ case. We plot the probability densities of the two zero-energy states in Fig. 4(b) and (c). As was pointed out earlier, the densities are mostly distributed along the block edges. However, we find no sign of fractionalization as in the earlier case, even though we exhaust all linear combinations of the states, which only lead to the probability density transfer between the corners and the edges (somewhat like breathing).

We further study the IQH states with $\nu>2$. For example, four Dirac points with Berry flux $\pi$ and one additional Dirac point with flux $-\pi$ can appear for $\nu=3$, and four Dirac points with Berry flux $\pi$ can appear for $\nu=4$, which are consistent with the $C_{4}$ lattice symmetry in the Hoftadter lattice model. In general, the number of the Dirac cones can be affected by the reconstruction of the entanglement spectrum, but the associated Berry flux cannot. In other words, the Berry flux for the ground state wave function of the entanglement Hamiltonian provides a topological $\mathbb{Z}$ classification of the transitions from the original IQH states to the trivial one.

Conclusion and discussion.- We have shown that the critical theory between the topological state and the corresponding trivial state can emerge in the entanglement spectrum of the non-trivial state with a symmetric checkerboard bipartition. The bipartition generates a quantum network with gapless bulk excitations. The critical theories for topologically different systems can be distinguished by the behavior of the low-energy entanglement spectrum and its degenerate zero-energy states. 
The IQH states to insulator transitions we discuss here are in the clean limit, which may be tuned by an additional periodic potential[24]. Distinct effects of quenched disorder may be considered separately.

Our results suggest that the Chern index characterizing the IQH states corresponds faithfully to the Berry flux of the ground state wave function of the entanglement Hamiltonian. The emergent particle-hole symmetry in the entanglement spectrum does not change the characterization of the critical theory, even though it manifests in the degenerate zero-energy states in the entanglement Hamiltonian. The bulk entanglement spectra for both $\nu=1$ and $\nu=2$ IQH states are gapless. This contrasts to the claim in Ref. [14] and the difference may be attributed to the additional symmetries in their model Hamiltonian unnecessary for the IQH states.

Remarkably, the ground state wave function of the topological bulk theory encodes not only the boundary theory [8], but also the critical theory for its transition to a trivial insulator with the same symmetry, both of which can be derived from the ground state entanglement spectrum under different types of bipartitions [14, 2527]. Therefore, we can expect a generic triangular correspondence among the bulk topological theories, the gapless edge theories, and the bulk critical theories. The edge theories is the critical theories spatially confined between the topological phases and the trivial insulating phases, while the percolating quantum networks of the edge modes establish the critical theories for the transitions of the topological phases to the trivial phases.

One of the authors (XW) would like to thank M.-C. Chung for many valuable discussions on the entanglement spectrum in free lattice models. The authors acknowledge the support of the 973 Program under Project No. 2012CB927404 and of NSF-China through the grants No. 20121302227 and No. 11174246.

[1] K. S. Novoselov et al., Nature 438, 197 (2005).

[2] Y. Zhang, J. W. Tan, H. L. Stormer, and P. Kim, Nature 438, 201 (2005).
[3] D. J. Thouless, M. Kohmoto, M. P. Nightingale, and M. den Nijs, Phys. Rev. Lett. 49, 405 (1982).

[4] K. von Klitzing, G. Dorda, and M. Pepper, Phys. Rev. Lett. 45, 494 (1980).

[5] F. D. M. Haldane, Phys. Rev. Lett. 61, 2015 (1988).

[6] A. W. W. Ludwig, M. P. A Fisher, R. Shankar, and G. Grinstein, Phys. Rev. B 50, 7526 (1994).

[7] K. S. Novoselov, et al., Nature Phys. 2, 177 (2006).

[8] H. Li and F. D. M. Haldane, Phys. Rev. Lett. 101, 010504 (2008).

[9] R. Thomale, A. Sterdyniak, N. Regnault, and B. A. Bernevig, Phys. Rev. Lett. 104, 180502 (2010).

[10] A. Chandran, M. Hermanns, N. Regunault, and B. A. Bernevig, Phys. Rev. B 84, 205136 (2011).

[11] X. L. Qi, H. Katsura, and A. W. W. Ludwig, Phys. Rev. Lett. 108, 196402 (2012).

[12] V. Alba, M. Haque, and A. M. Lauchli, Phys. Rev. Lett. 108, 227201 (2012).

[13] X. Chen, F. Wang, Y. M. Lu, and D. H. Lee, Nucl. Phys. B 873, 248 (2013).

[14] T. H. Hsieh and L. Fu, Phys. Rev. Lett. 113, 106801 (2014).

[15] A. H. Castro Neto, F. Guinea, N. M. R. Peres, K. S. Novoselov, and A. K. Geim, Rev. Mod. Phys. 81, 109 (2009).

[16] E. McCann and V. I. Fal'ko, Phys. Rev. Lett. 96, 086805 (2006).

[17] D. R. Hofstadter, Phys. Rev. B 14, 2239 (1976).

[18] A. P. Schnyder et al., Phys. Rev. B 78, 195125 (2008); A. Kitaev, AIP Conf. Proc. 1134, 22 (2009).

[19] For a review, see I. Peschel and V. Eisler, J. Phys. A: Math. Theor. 42, 504003 (2009).

[20] J. T. Chalker and P. D. Coddington, J. Phys. C 21, 2665 (1988).

[21] C. M. Ho and J. T. Chalker, Phys. Rev. B 54, 8708 (1996).

[22] R. Jackiw, Phys. Rev. D 29, 2375 (1984).

[23] K. Sun, H. Yao, E. Fradkin, and S. A. Kivelson, Phys. Rev. Lett. 103, 046811 (2009).

[24] X. G. Wen and Y. S. Wu, Phys. Rev. Lett. 70, 1501 (1993); W. Chen, M. P. A. Fisher, and Y. S. Wu, Phys. Rev. B 4813749 (1993).

[25] W. J. Rao, X. Wan, and G. M. Zhang, Phys. Rev. B 90, 075151 (2014).

[26] T. H. Hsieh, L. Fu, and X. L. Qi, Phys. Rev. B 90, 085137 (2014).

[27] R. A. Santos, arXiv:1408.1716 (unpublished). 